\title{
Effects of hepatic glycogen on food intake and glucose homeostasis are mediated by the vagus nerve in mice
}

\author{
Iliana López-Soldado ${ }^{1,2}$ • Rebeca Fuentes-Romero ${ }^{1}$. Jordi Duran ${ }^{1,2}$ • \\ Joan J. Guinovart ${ }^{1,2,3}$
}

Received: 12 December 2016/Accepted: 14 February 2017 / Published online: 15 March 2017

(C) Springer-Verlag Berlin Heidelberg 2017

\begin{abstract}
Aims/hypothesis Liver glycogen plays a key role in regulating food intake and blood glucose. Mice that accumulate large amounts of this polysaccharide in the liver are protected from high-fat diet (HFD)-induced obesity by reduced food intake. Furthermore, these animals show reversal of the glucose intolerance and hyperinsulinaemia caused by the HFD. The aim of this study was to examine the involvement of the hepatic branch of the vagus nerve in regulating food intake and glucose homeostasis in this model.

Methods We performed hepatic branch vagotomy (HBV) or a sham operation on mice overexpressing protein targeting to glycogen $\left(P t g^{\mathrm{OE}}\right)$. Starting 1 week after surgery, mice were fed an HFD for 10 weeks.

Results HBV did not alter liver glycogen or ATP levels, thereby indicating that this procedure does not interfere with hepatic energy balance. However, HBV reversed the effect of glycogen accumulation on food intake. In wild-type mice, HBV led to a significant reduction in body weight without a change in food intake. Consistent with their body weight reduction, these animals had decreased fat deposition,
\end{abstract}

Iliana López-Soldado and Rebeca Fuentes-Romero contributed equally to this study.

Joan J. Guinovart

guinovart@irbbarcelona.org

1 Institute for Research in Biomedicine (IRB Barcelona), Barcelona Institute of Science and Technology (BIST), Baldiri i Reixac 10, 08028 Barcelona, Spain

2 Centro de Investigación Biomédica en Red de Diabetes y Enfermedades Metabólicas Asociadas (CIBERDEM), Madrid, Spain

3 Department of Biochemistry and Molecular Biomedicine, University of Barcelona, Barcelona, Spain adipocyte size, and insulin and leptin levels, together with increased energy expenditure. Ptg ${ }^{\mathrm{OE}}$ mice showed an increase in energy expenditure and glucose oxidation, and these differences were abolished by HBV. Moreover, Ptg $^{\mathrm{OE}}$ mice showed an improvement in HFDinduced glucose intolerance, which was suppressed by HBV.

Conclusions/interpretation Our results demonstrate that the regulation of food intake and glucose homeostasis by liver glycogen is dependent on the hepatic branch of the vagus nerve.

Keywords Diabetes · Food intake - Glucose homeostasis · Glycogen $\cdot$ Hepatic vagus nerve $\cdot$ High-fat diet $\cdot$ Liver . Metabolism · Mice
Abbreviations
CNS Central nervous system
HBV Hepatic branch vagotomy
HFD High-fat diet
PTG Protein targeting to glycogen

\section{Introduction}

The main cause of obesity and overweight is an imbalance between energy consumption and energy expenditure. Several mechanisms are involved in maintaining energy homeostasis. Secreted humoral factors convey information about energy storage from adipose tissue to the central nervous system (CNS). In addition to these factors, neuronal signals play key roles in energy homeostasis [1-4]. Hepatic 
signals are postulated to be transmitted to the CNS by the vagus nerve because vagal activity is increased by portal or jejunal infusion of lipids [5].

Several reports have highlighted the contribution of the vagus nerve to regulating food intake. The stimulation of feeding by 2,5-anhydro-D-mannitol was shown to be dependent on this nerve [6]. Hepatic branch vagotomy (HBV) blocks the stimulation of satiety by a variety of fuels [7]. In addition, the hepatic vagus nerve mediates fat-induced inhibition of diabetic hyperphagia by enabling the restoration of hypothalamic neuropeptide expression [8,9]. Furthermore, inhibition of fatty acid oxidation by mercaptoacetate increases food intake, and this effect is abolished by HBV [10]. Furthermore, HBV has been shown to attenuate the feeding response to peripheral 2-deoxyglucose [11-13].

The search for a metabolic sensor that regulates food intake has been actively pursued for many years. Mayer [14] developed the 'glucostatic' theory of feeding behaviour, proposing that changes in glucose utilisation rates regulate hunger and satiety. Russek $[15,16]$ later put forward a 'hepatostatic' theory, which was further refined by Flatt into the 'glycogenostatic' model [17], in which hepatic glycogen stores act as a glucoreceptor involved in the regulation of food intake. Langhans and colleagues described a relationship between hepatic glycogen levels and spontaneous eating [18]. In the following years, a large body of experimental evidence established an association between the size of glycogen stores in the liver and the regulation of food intake [19-23]. We recently showed that mice that accumulate high levels of hepatic glycogen are protected from high-fat diet (HFD)-induced obesity as a result of reduced food intake [23]. We propose that increased liver glycogen stores contribute to decreased appetite and adiposity, and that this effect is probably triggered by signals from the liver that are transmitted to the brain via the hepatic vagus nerve. To test this hypothesis, we studied the effects of HBV in mice overexpressing protein targeting to glycogen $\left(\mathrm{Ptg}^{\mathrm{OE}}\right)$ and control mice fed a HFD.

\section{Methods}

Animals and diets All procedures were approved by the Barcelona Science Park Animal Experimentation Committee and carried out in accordance with the European Community Council Directive and the National Institute of Health guidelines for the care and use of laboratory animals. Mice that overexpress Ptg (also known as Ppp1r3c) in the liver $\left(P \operatorname{tg}^{\mathrm{OE}}\right)$ were generated as previously described [23]. Male $P t g{ }^{\mathrm{OE}}$ and control mice were housed with free access to water and a standard chow diet (Envigo, Indianapolis, IN, USA) before surgery. Starting 1 week after surgery, mice were fed a HFD (45\% fat content; D12451 Formula, Research Diets, New Brunswick, NJ, USA) for 10 weeks.

Vagus nerve denervation and sham operation The common hepatic branch of the vagus nerve of 6-week-old mice was cut. Briefly, mice were anaesthetised with isoflurane (2-3\% with $\mathrm{O}_{2}$ ) and placed on a surgical heating pad. After the abdominal region had been shaved and disinfected, laparotomy was performed. The hepatic lobules were gently displaced in a dorsal anterior direction. The ligaments surrounding the liver were removed to expose the oesophagus and stomach. The hepatic branch of the vagus nerve was then separated from the left side of the liver. Care was taken not to damage the dorsal and ventral parts of the vagus that innervate the stomach and abdominal tissue. The abdominal wall and skin were then closed. Buprenorphine was administered, together with $0.9 \% \mathrm{NaCl}$, to compensate for intraoperative fluid loss. Control mice underwent a sham operation, i.e. we repeated the surgery without cutting the nerves or connective tissue. After surgery, mice were housed individually for the remainder of the experiment. After 10 weeks on the HFD, mice were either fed or fasted for $16 \mathrm{~h}$ before being killed. Tissues were collected and stored at $-80^{\circ} \mathrm{C}$ until analysis or preserved in $10 \%$ phosphate-buffered formalin for histological analysis.

Blood and liver biochemical analysis Hepatic glycogen was quantified as previously described [24]. The intracellular concentration of ATP was measured in perchloric acid extracts of livers by HPLC. Approximately $50 \mathrm{mg}$ frozen tissue was homogenised in $500 \mu 110 \% \mathrm{HClO}_{4}$. Samples were clarified at $3000 \mathrm{~g}$ at $4^{\circ} \mathrm{C}$ for $15 \mathrm{~min}$ and supernatants were neutralised with $0.5 \mathrm{M} \mathrm{K}_{2} \mathrm{CO}_{3}$. Samples were placed on ice for $5 \mathrm{~min}$ and then centrifuged at $3000 \mathrm{~g}$ at $4{ }^{\circ} \mathrm{C}$ for $10 \mathrm{~min}$. ATP analysis was performed using a Brisa LC2 C18 column $(4.6 \times 150 \mathrm{~mm}$, $3 \mu \mathrm{m}$ particle size) interfaced with a photodiode array detector $(260 \mathrm{~nm})$ and a constant flow rate of $0.6 \mathrm{ml} / \mathrm{min}$. ATP was separated using a mobile phase gradient (70:30 ratio of eluent A [500 mmol/l $\mathrm{KH}_{2} \mathrm{PO}_{4}, 4 \mathrm{mmol} / \mathrm{l}\left(\mathrm{C}_{4} \mathrm{H}_{9}\right)_{4} \mathrm{~N}\left(\mathrm{HSO}_{4}\right)$, $\mathrm{pH}$ 6]/methanol) for $37 \mathrm{~min}$ and quantified against known standards in eluent A. Liver lactate was measured in perchloric acid extracts using a commercial spectrophotometric kit (Horiba, ABX, Montpellier, France). Blood glucose levels were measured using a glucometer (Ascensia Breeze 2, Bayer Healthcare, Leverkusen, Germany). Plasma insulin and leptin were analysed by ELISA (Crystal Chem, Downers Grove, IL, USA).

In vivo metabolic analysis Glucose tolerance tests were performed as previously described [23]. Indirect calorimetry was performed using an eight-chamber Oxymax system (Columbus Instruments International, Columbus, OH, USA) to measure energy expenditure, calculated from oxygen consumption and carbon dioxide production, as previously 
described [23]. In order to monitor food intake, mice were housed individually and food was measured daily.

RNA preparations and quantitative PCR Total RNA was isolated from frozen liver samples using Trizol reagent (Life Technologies, Carlsbad, CA, USA) and purified with an RNeasy Mini Kit (Qiagen, Hilden, Germany) and treated with DNase I (Qiagen) to degrade genomic DNA. Reverse transcription was then performed using qScript cDNA Synthesis Kit (Quanta Biosciences, Beverly, MA, USA). Quantitative (q)PCR was performed using a Quantstudio 6 Flex (Applied Biosystems, Foster City, CA, USA). The following TaqMan primer and probe sets (Applied Biosystems) were used for qPCR: Pklr (Mm 00443090_m1), Ppia (Mm02342429_g1). The latter was used as a reference gene.

Histology A portion of subcutaneous fat was H\&E stained and adipocyte size was measured as previously described [23].

Statistical analysis Data are expressed as means \pm SEM. Groups were compared using two-way or three-way ANOVA with post hoc Tukey tests. A $p$ value of $<0.05$ was considered significant.

\section{Results}

Liver glycogen, ATP and hepatic lactate levels are unaffected by $\mathrm{HBV}$ Ptg $^{\mathrm{OE}}$ and wild-type mice underwent HBV or a sham operation, followed by a HFD for 10 weeks.
First, we measured the glycogen content of livers of the different experimental groups. In the fed state, $\mathrm{Ptg}^{\mathrm{OE}}$ mice showed a twofold increase in liver glycogen compared with control animals (Fig. 1a). After overnight fasting, control mice had lower hepatic glycogen levels, while $\mathrm{Ptg}^{\mathrm{OE}}$ mice maintained similar levels as in the fed state (Fig. 1a). Furthermore, there were no differences in liver glycogen levels between sham-operated and HBV groups, indicating that hepatic denervation did not alter the liver glycogen content (Fig. 1a).

To examine whether HBV affects hepatic energy status, we measured ATP levels in the liver. The hepatic ATP content was higher in $\mathrm{Ptg}^{\mathrm{OE}}$ mice compared with control mice in the fed state. ATP levels decreased significantly in control mice in the fasted state compared with the fed state; in contrast, ATP levels in the livers of fasted $\mathrm{Ptg}^{\mathrm{OE}}$ mice were similar to those in the fed state. Remarkably, we did not observe changes in the level of ATP between HBV and sham-operated groups for either genotype (Fig. 1b). Levels of liver lactate increased in $\mathrm{Ptg}^{\mathrm{OE}}$ mice compared with control mice in the fasted state (Fig. 1c). The mRNA levels of pyruvate kinase in the livers of Ptg $^{\mathrm{OE}}$ mice were also higher under fasting conditions compared with those of control mice (Fig. 1d). There were no differences in hepatic lactate levels or mRNA levels of Pklr between sham-operated and HBV groups (Fig. 1c, d).

HBV reverses the effect of PTG overexpression on HFD food intake To study whether HBV has an effect on food ingestion, we measured food intake. Consistent with our previous report [23], sham-Ptg ${ }^{\mathrm{OE}}$ mice ate less compared with
Fig. 1 Liver glycogen, ATP and lactate levels are unaffected by HBV. Control (white bars) and $\operatorname{Ptg}^{\mathrm{OE}}$ (black bars) mice underwent HBV or sham surgery (Sham). After surgery, mice were fed an HFD for 10 weeks and then killed after normal feeding (Fed) or $16 \mathrm{~h}$ fasting (Fasted). Liver glycogen content (a), liver ATP content (b), liver lactate levels (c) and liver Pklr mRNA (d) under fed conditions and fasting conditions. ${ }^{*} p<0.05$ vs control mice subjected to the same surgical procedures; ${ }^{\dagger} p<0.05$, fed vs fasted mice a

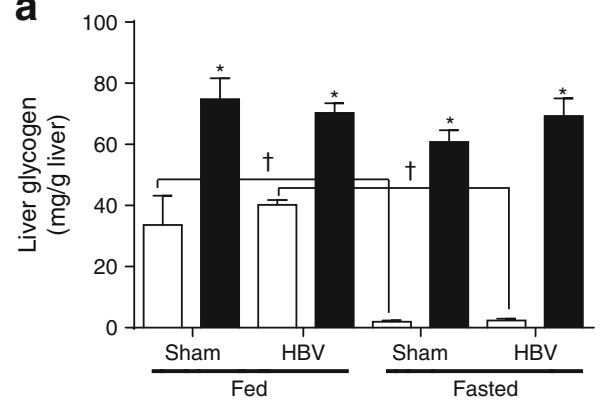

C

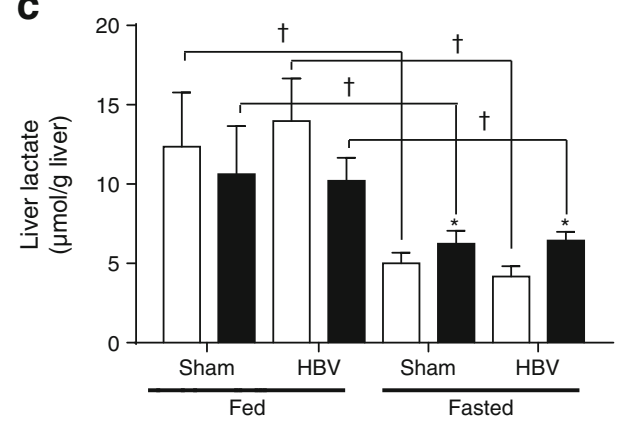

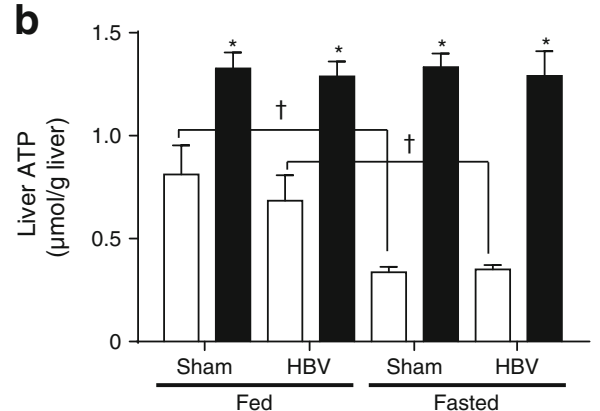

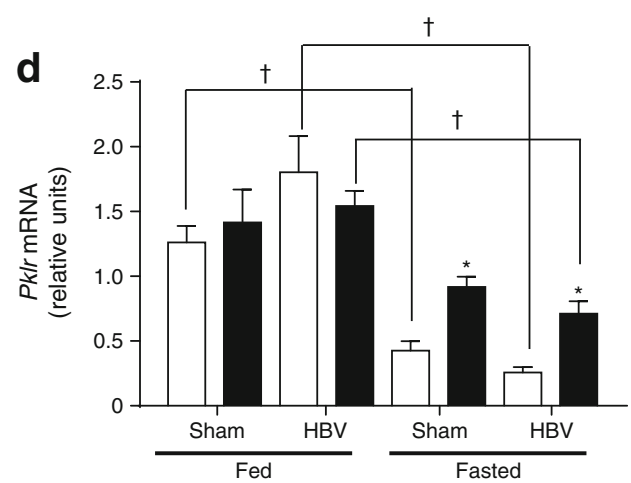


sham-control animals (Fig. 2a) when fed a HFD. However, HBV-Ptg ${ }^{\mathrm{OE}}$ mice had a similar food intake to that of HBV-control animals, indicating that HBV reversed the effect of PTG overexpression on food intake. In control mice, HBV did not have any effect on food intake. Next, we studied the contribution of the hepatic vagus nerve to body weight and fat weight in control and $\mathrm{Ptg}^{\mathrm{OE}}$ mice. $P \operatorname{tg}^{\mathrm{OE}}$ mice had a lower body weight than control mice when the hepatic vagus nerve was intact. However, this difference was not observed between HBV-Ptg ${ }^{\mathrm{OE}}$ and HBV-control mice, which had similar body weights (Fig. 2b). Body weights of sham-Ptg ${ }^{\mathrm{OE}}$ and HBV-Ptg ${ }^{\mathrm{OE}}$ mice were also similar (Fig. 2b). Furthermore, HBV caused a reduction in body weight in control mice (Fig. 2b). Consistent with their reduced body weight, HBV-control mice had a decrease in subcutaneous fat weight, epididymal fat weight and adipocyte size compared with sham-control mice (Fig. 2c-e). As previously described [23], sham-Ptg ${ }^{\mathrm{OE}}$ mice had a lower subcutaneous fat weight and a lower adipocyte size compared with sham-controls (Fig. 2c, e). In contrast, HBV blocked the decrease in adipose tissue weight and adipocyte size seen in $\operatorname{Ptg}^{\mathrm{OE}}$ mice. These observations thus indicate that the vagus nerve mediates the remote effects of hepatic glycogen content.

HBV decreases glucose oxidation in $\boldsymbol{P t g}^{\mathbf{O E}}$ mice We next investigated whether HBV has an impact on energy expenditure. In control mice, HBV led to a significant increase in energy expenditure throughout the light and dark phases. $\mathrm{Ptg}^{\mathrm{OE}}$ mice showed greater energy expenditure during the light and dark phases when the vagus nerve was not ablated (Fig. 3a). This difference in energy expenditure was abolished by HBV (Fig. 3a). As previously described [23], Ptg $^{\mathrm{OE}}$ animals showed an increase in the respiratory exchange ratio, indicating that they used more carbohydrates as an energy source compared with the control group. This increase was reversed by HBV (Fig. 3b). Consistent with this finding, glucose oxidation was increased in $P \operatorname{tg}{ }^{\mathrm{OE}}$ mice compared with control mice, and HBV abolished this effect (Fig. 3c). No
Fig. 2 The hepatic vagus nerve is required to mediate the suppressive effect of food intake in $P t{ }^{\mathrm{OE}}$ mice. Control and Ptg ${ }^{\mathrm{OE}}$ mice underwent $\mathrm{HBV}$ or sham surgery (Sham). After surgery, mice were fed an HFD for 10 weeks. (a) Food intake in control (white bars) and Ptg ${ }^{\mathrm{OE}}$ (black bars) mice. (b) Mean body weight \pm SEM of control-sham (white circles), tg $^{\mathrm{OE}}$-sham (black circles), control-HBV (blue circles) and $\mathrm{Ptg}^{\mathrm{OE}}-\mathrm{HBV}$ (red triangles) mice. (c, d) Subcutaneous (c) and epididymal (d) fat weight in control (white bars) and Ptg $^{\mathrm{OE}}$ (black bars) mice. (e) H\&E stained subcutaneous adipose tissue (scale bars, $100 \mu \mathrm{m})$; the histogram shows adipocyte size in subcutaneous adipose tissue in control (white bars) and $P t^{\mathrm{OE}}$ (black bars) mice. ${ }^{*} p<0.05$ vs control mice subjected to the same surgical procedure; ${ }^{\dagger} p<0.05$, sham-operated vs HBV mice of the same genotype
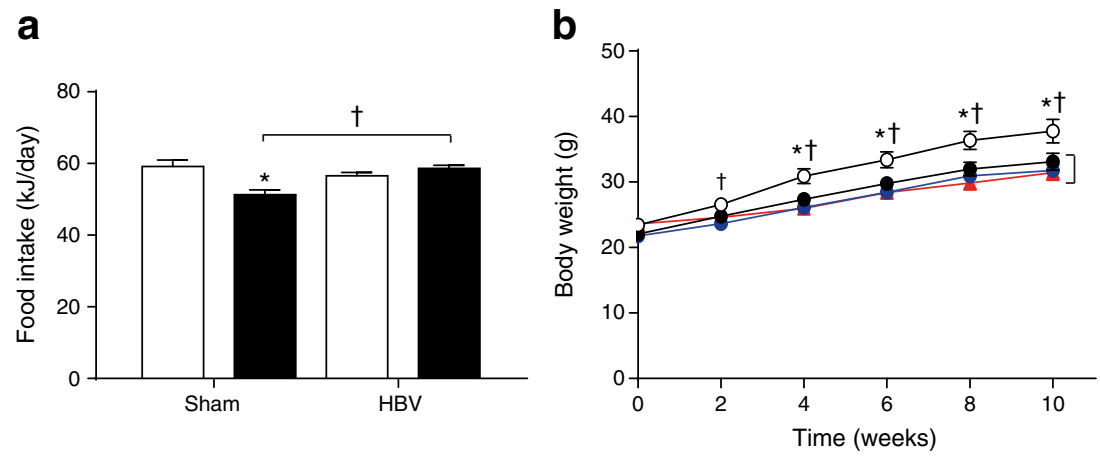

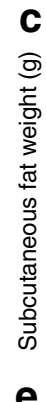

d
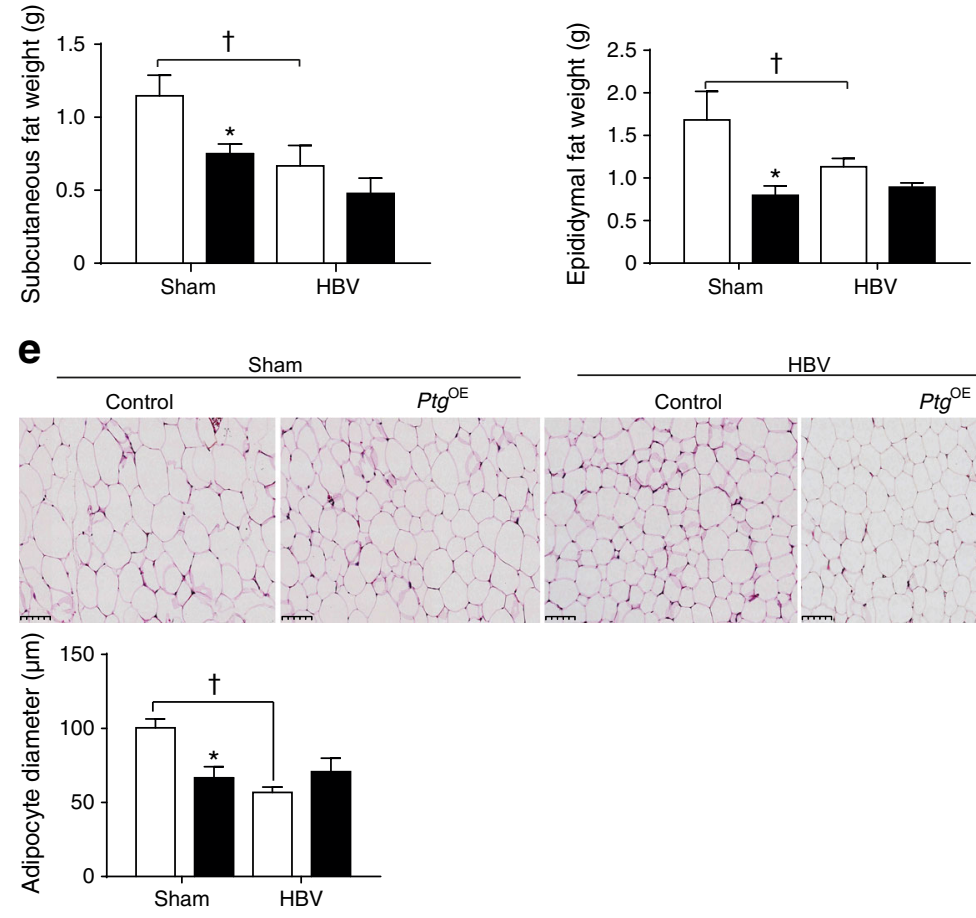

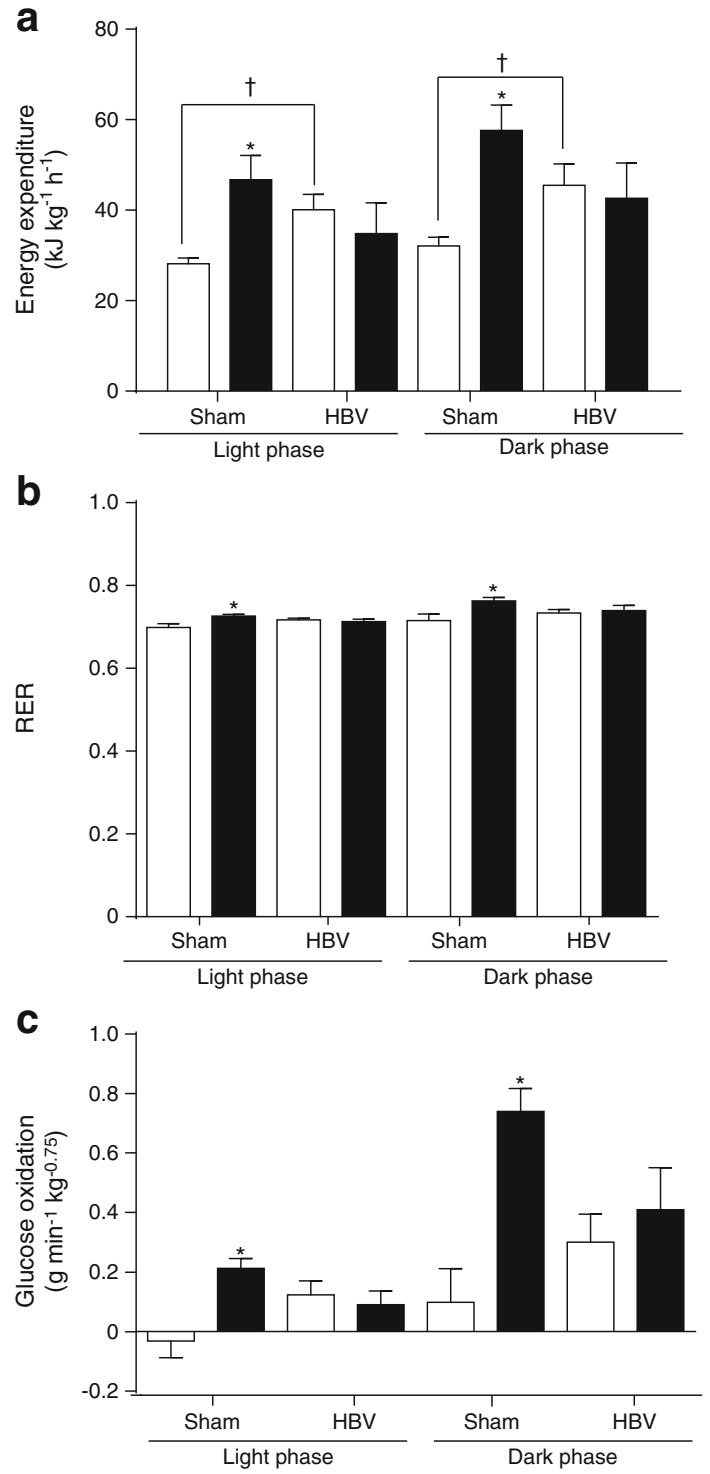

Fig. 3 HBV decreased glucose oxidation in $\operatorname{Ptg}^{\mathrm{OE}}$ mice. Control (white bars) and $P^{\mathrm{OE}}$ (black bars) mice underwent HBV or sham surgery (Sham). After surgery, mice were fed an HFD for 10 weeks. (a-c) Resting energy expenditure (a), respiratory exchange ratio (RER) (b) and glucose oxidation rate (c) during the light and dark phases. $* p<0.05$ vs control mice subjected to the same surgical procedure; ${ }^{\dagger} p<0.05$, sham-operated vs HBV mice of the same genotype

differences in physical activity were found between groups (data not shown).

HBV alters blood metabolite levels Consistent with our previous report [23], sham-Ptg ${ }^{\mathrm{OE}}$ mice had lower blood glucose levels and lower plasma insulin concentrations than sham-control animals when fed a HFD (Fig. 4a, b). Remarkably, in the fed state, Ptg $^{\mathrm{OE}}$ mice failed to reduce blood glucose and insulin levels when the vagus nerve was ablated (Fig. 4a, b). HBV had no effect on glycaemia (Fig. 4a) but led to a decrease in plasma insulin levels in control mice (Fig. 4b). Leptin serum levels were also significantly reduced in HBV-control mice compared with sham-control mice (Fig. 4c), and HBV blocked the decrease in serum leptin induced by hepatic glycogen (Fig. 4c).

The vagus nerve contributes to glucose homeostasis in Ptg $^{\mathbf{O E}}$ mice Ptg $^{\mathrm{OE}}$ mice showed improved glucose tolerance compared with controls (Fig. 5), and HBV abolished this effect (Fig. 5). These observations demonstrate that liver glycogen regulates glucose metabolism through the vagus nerve.

\section{Discussion}

Here, we studied the involvement of the hepatic branch of the vagus nerve in regulating food intake and glucose homeostasis in $P g^{\mathrm{OE}}$ mice, which accumulate high levels of glycogen in the liver. These mice showed reduced food intake and a reversal of glucose intolerance when fed a HFD [23]. We demonstrated that $\mathrm{HBV}$ abolishes the effect of glycogen accumulation on food intake. In contrast, HBV-control mice ate the same amount of food as sham-control animals when fed a HFD. This finding is in line with observations made in previous studies of rats [25, 26] and mice [27]. It is noteworthy that although both HBV groups and sham-control mice had similar food intakes, they did not weigh the same. This finding suggests that HBV causes a decrease in body weight and promotes resistance to diet-induced obesity. Thus, loss of the nerve connection itself had an impact on body weight independent of glycogen accumulation, as described by two recent studies in rats [28] and mice [27]. Consistent with their reduced body weight, our HBV mice had a decrease in subcutaneous fat mass, smaller adipocytes and lower levels of leptin in plasma. Similar results have been reported in various studies [2, 27-29]. Taken together, these observations reinforce the notion that $\mathrm{HBV}$ reduces body weight and induces resistance to diet-induced obesity.

In an attempt to explain the metabolic changes observed after HBV, we examined whole body energy expenditure. Sham-Ptg ${ }^{\mathrm{OE}}$ mice had an increase in energy expenditure, which, together with their lower food intake, could explain the lower body weight of this group. Energy expenditure also differed between HBV-control mice and sham-control mice, in accordance with the findings of Gao et al [27]. These authors reported an increase in energy expenditure during the dark phase in HBV mice compared with sham-operated mice, concluding that this increase may account for the reduction in body weight of the former. Moreover, $\mathrm{Ptg}^{\mathrm{OE}}$ mice oxidised more glucose than control mice, and HBV abolished this effect. These findings indicate that neuronal signals through the hepatic vagus nerve are also involved in regulating carbohydrate oxidation in these mice.

As previously described, PTG overexpression resulted in an increase in liver glycogen. Consistent with previous results [30], 
Fig. 4 Effect of HBV on blood metabolite levels. Control (white bars) and Ptg $^{\mathrm{OE}}$ (black bars) mice underwent HBV or sham surgery (Sham). After surgery, mice were fed an HFD for 10 weeks.

(a) Blood glucose, (b) plasma insulin and (c) plasma leptin concentrations were measured. $* p<0.05$ vs control mice subjected to the same surgical procedure; ${ }^{\dagger} p<0.05$, sham-operated vs HBV mice of the same genotype
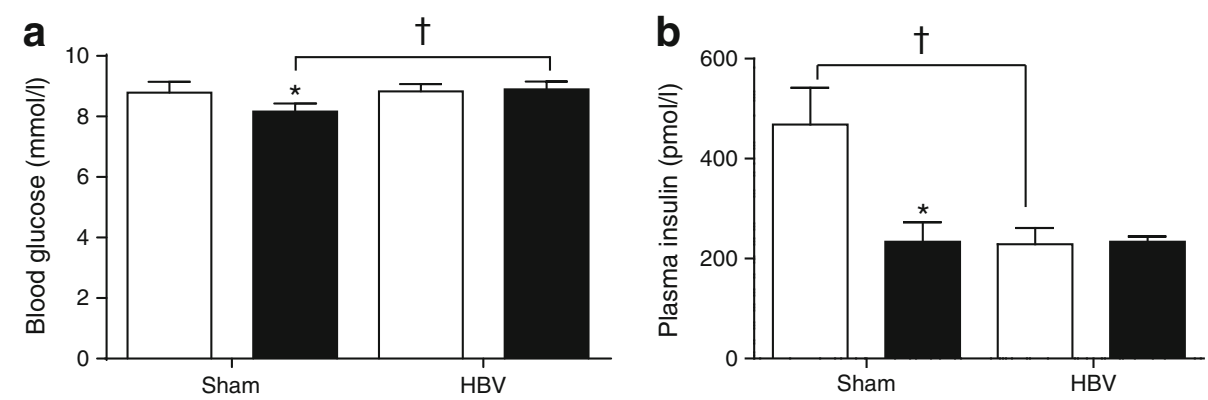

HBV did not affect liver glycogen levels. Moreover, our results demonstrated that $\operatorname{Ptg}^{\mathrm{OE}}$ mice fed a HFD had higher ATP levels compared with control mice, and that HBV did not interfere with hepatic ATP levels, as previously reported [8]. Fasted $P t g{ }^{\mathrm{OE}}$ mice activated glycolysis (indicated by the increase in lactate and $P k l r$ mRNA levels), suggesting that this mechanism could be one way in which $P \operatorname{tg}{ }^{\mathrm{OE}}$ mice maintain ATP levels in the liver. ATP is a key metabolite that triggers food intake through the vagus nerve $[6,31]$. It has been hypothesised that an increasing ATP concentration in the hepatocytes inhibits the firing of hepatic vagal afferents, inhibits activation of the nucleus tractus solitarius, and suppresses the appetite [32]. Since sectioning the hepatic vagus eliminates most of these responses, it is presumed that ATP levels in the hepatocyte are conveyed to the CNS via vagal nerves. Therefore, liver glycogen, through maintaining hepatic ATP levels, contributed to decreasing appetite and this effect was triggered by the vagus nerve. In rats, it has been shown that sensory

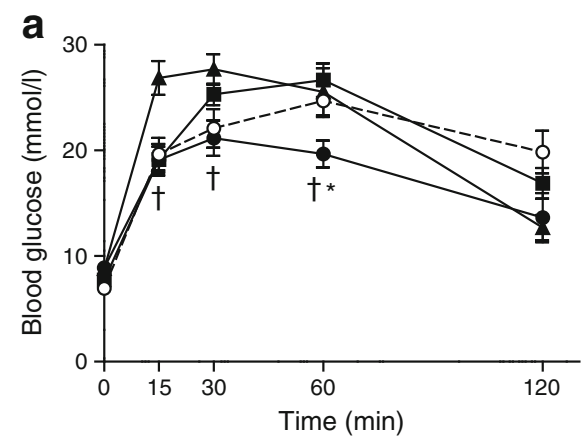

Fig. 5 The vagus nerve contributes to glucose homeostasis in Ptg $^{\mathrm{OE}}$ mice. Control and $\operatorname{Ptg}^{\mathrm{OE}}$ mice underwent HBV or sham surgery. After surgery, mice were fed an HFD for 10 weeks. (a) GTT curve for glucose after 8 weeks on an HFD for control-sham (white circles and dashed line), $\mathrm{Ptg}^{\mathrm{OE}}$-sham (black circles and solid line), control-HBV (black squares vagal fibres do not provide substantial direct innervation of hepatocytes, but rather with portal triads in proximity to branches of the hepatic artery, portal vein and bile ducts [33]. However, neuroanatomical data for the vagus in mice are limited. In fact, several reports in mice describe metabolic information in the liver being transmitted to the brain via the hepatic vagus [2, 34-36]. Nonetheless, we cannot exclude the possibility that the mechanism described in our study depends on sensors located in the portal region. Another factor that could complicate the interpretation of the data is that cutting the common hepatic vagal branch denervates not only the liver but also parts of the gastrointestinal tract [33]. Despite these neuroanatomical difficulties, the evidence presented here highlight the liver as a powerful metabolic sensor, and a crucial link in the brain regulation of energy metabolism [37].

There is a link between the CNS and hepatic glucose production [38] that involves the hepatic branch of the vagus;

\section{b}

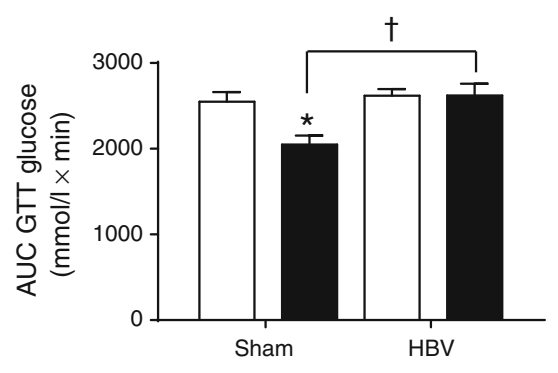

and solid line) and Ptg $^{\mathrm{OE}}$-HBV (black triangles and solid line) (b) AUC for glucose in control (white bars) and $\operatorname{Ptg}^{\mathrm{OE}}$ (black bars) mice. ${ }^{*} p<0.05$ vs control mice subjected to the same surgical procedure; ${ }^{\dagger} p<0.05$, sham-operated vs HBV mice of the same genotype 
however, this link is efferent, not afferent [4]. Moreover, intact vagal afferent fibres are required for glucocorticoid-induced glucose intolerance [34]. $\mathrm{Ptg}^{\mathrm{OE}}$ mice showed an improvement in HFD-induced glucose intolerance, which was abolished by HBV. This observation indicates that an intact vagus nerve is required for HFD-induced glucose intolerance. This notion is consistent with a recent study in dogs indicating that hepatic glycogen regulates hypoglycaemic counter-regulation via a liver-brain axis [39]. In our model system, HBV lowered insulin without changing glucose levels, indicating increased insulin sensitivity. In rats, HBV has been reported to induce insulin resistance [40, 41]. However, in dexamethasone-treated mice, HBV resulted in greater insulin responsiveness during insulin tolerance testing compared with sham surgery, and this effect persisted for at least 3 months after the surgery [34]. Thus, in models in which insulin resistance is already present, for example, as induced by glucocorticoids [34] or in young animals fed a HFD [29], HBV seems to increase insulin sensitivity.

Rather than being a direct consequence of liver denervation [8], our findings support the hypothesis that a neural signal to the CNS is involved in the inhibition of food intake caused by liver glycogen accumulation. Therefore, we conclude that liver glycogen accumulation triggers a liver-brain neuronal network to regulate food intake and glucose homeostasis.

Acknowledgements The authors thank M. García Rocha, A. Adrover, E. Veza, N. Plana and A. Berenguer, all from the Institute of Research in Biomedicine (IRB Barcelona) and staff of the Scientific and Technological Services of the University of Barcelona for technical assistance.

Data availability The data are available on request from the authors.

Funding This study was supported by grants from the Spanish MINECO (SAF2014-54525-P), Fundació Marató de TV3 (201613-10) and CIBERDEM (Instituto de Salud Carlos III, Spanish Ministry of Science and Innovation). None of the supporting agencies had any role in performing the work or in writing the manuscript.

Duality of interest statement The authors declare that there is no duality of interest associated with this manuscript.

Contribution statement IL-S, JJG and RF-R conceived the study and designed all experiments; JJG directed the study; IL-S and RF-R analysed the data; IL-S, JD and JJG interpreted the data; IL-S and RF-R wrote the manuscript. All authors approved the final version of the manuscript to be published. JJG is responsible for the integrity of the work as a whole.

\section{References}

1. Izumida Y, Yahagi N, Takeuchi Y et al (2013) Glycogen shortage during fasting triggers liver-brain-adipose neurocircuitry to facilitate fat utilization. Nat Commun 4:2316

2. Uno K, Katagiri H, Yamada T et al (2006) Neuronal pathway from the liver modulates energy expenditure and systemic insulin sensitivity. Science 312:1656-1659
3. Yamada T, Katagiri H, Ishigaki Y et al (2006) Signals from intraabdominal fat modulate insulin and leptin sensitivity through different mechanisms: neuronal involvement in food-intake regulation. Cell Metab 3:223-229

4. Lam TK, Schwartz GJ, Rossetti L (2005) Hypothalamic sensing of fatty acids. Nat Neurosci 8:579-584

5. Randich A, Spraggins DS, Cox JE, Meller ST, Kelm GR (2001) Jejunal or portal vein infusions of lipids increase hepatic vagal afferent activity. Neuroreport 12:3101-3105

6. Ritter S, Dinh TT, Friedman MI (1994) Induction of Fos-like immunoreactivity (Fos-li) and stimulation of feeding by 2,5-anhydroD-mannitol (2,5-AM) require the vagus nerve. Brain Res 646:53-64

7. Langhans W, Egli G, Scharrer E (1985) Selective hepatic vagotomy eliminates the hypophagic effect of different metabolites. J Auton Nerv Syst 13:255-262

8. la Fleur SE, Ji H, Manalo SL, Friedman MI, Dallman MF (2003) The hepatic vagus mediates fat-induced inhibition of diabetic hyperphagia. Diabetes 52:2321-2330

9. la Fleur SE, Manalo SL, Roy M, Houshyar H, Dallman MF (2005) Hepatic vagotomy alters limbic and hypothalamic neuropeptide responses to insulin-dependent diabetes and voluntary lard ingestion. Eur J Neurosci 21:2733-2742

10. Langhans W, Scharrer E (1987) Evidence for a vagally mediated satiety signal derived from hepatic fatty acid oxidation. J Auton Nerv Syst 18:13-18

11. Delprete E, Scharrer E (1992) Effects of 2-deoxy-D-glucose on food intake of rats are affected by diet composition. Physiol Behav 51: 951-956

12. Del Prete E, Scharrer E (1994) Circadian effects of hepatic branch vagotomy on the feeding response to 2-deoxy-D-glucose in rats. J Auton Nerv Syst 46:27-36

13. Booth DA (1972) Modulation of the feeding response to peripheral insulin, 2-deoxyglucose or 3-O-methyl glucose injection. Physiol Behav 8:1069-1076

14. Mayer J (1955) Regulation of energy intake and the body weight: the glucostatic theory and the lipostatic hypothesis. Ann N Y Acad Sci 63:15-43

15. Russek M (1971) Hepatic receptors and the neurophysiological mechanisms controlling feeding behavior. Neurosci Res 4:213-282

16. Russek M (1963) Participation of hepatic glucoreceptors in the control of intake of food. Nature 197:79-80

17. Flatt JP (1996) Carbohydrate balance and body-weight regulation. Proc Nutr Soc 55:449-465

18. Langhans W, Geary N, Scharrer E (1982) Liver glycogen content decreases during meals in rats. Am J Phys 243:R450-R453

19. Emery PW, Carpenter TT, Obeid OA (1993) Alterations in postprandial glycogen and lipid synthesis in cachectic tumor-bearing rats. Nutr Cancer 20:231-240

20. Ros S, Garcia-Rocha M, Calbo J, Guinovart JJ (2011) Restoration of hepatic glycogen deposition reduces hyperglycaemia, hyperphagia and gluconeogenic enzymes in a streptozotocin-induced model of diabetes in rats. Diabetologia 54:2639-2648

21. Obeid OA, Powell-Tuck J, Emery PW (2000) The postprandial rates of glycogen and lipid synthesis of lean and obese female Zucker rats. Int J Obes Relat Metab Disord 24:508-513

22. Yang R, Newgard CB (2003) Hepatic expression of a targeting subunit of protein phosphatase-1 in streptozotocin-diabetic rats reverses hyperglycemia and hyperphagia despite depressed glucokinase expression. J Biol Chem 278:23418-23425

23. Lopez-Soldado I, Zafra D, Duran J, Adrover A, Calbo J, Guinovart JJ (2015) Liver glycogen reduces food intake and attenuates obesity in a high-fat diet-fed mouse model. Diabetes 64:796-807

24. Garcia-Rocha M, Roca A, De La Iglesia N et al (2001) Intracellular distribution of glycogen synthase and glycogen in primary cultured rat hepatocytes. Biochem J 357:17-24 
25. Bellinger LL, Williams FE (1988) The effect of liver denervation on the consumption of various diets by rats. Physiol Behav 43:527-531

26. Friedman MI, Sawchenko PE (1984) Evidence for hepatic involvement in control of ad libitum food intake in rats. Am J Phys 247: R106-R113

27. Gao X, van der Veen JN, Zhu L et al (2015) Vagus nerve contributes to the development of steatohepatitis and obesity in phosphatidylethanolamine $N$-methyltransferase deficient mice. J Hepatol 62: 913-920

28. Stearns AT, Balakrishnan A, Radmanesh A, Ashley SW, Rhoads DB, Tavakkolizadeh A (2012) Relative contributions of afferent vagal fibers to resistance to diet-induced obesity. Dig Dis Sci 57: $1281-1290$

29. Barella LF, Miranda RA, Franco CC et al (2015) Vagus nerve contributes to metabolic syndrome in high-fat diet-fed young and adult rats. Exp Physiol 100:57-68

30. Lavoie JM, Lord M, Paulin A (1988) Effect of selective hepatic vagotomy on plasma FFA levels in resting and exercising rats. Am J Phys 254:R602-R606

31. Grill HJ, Friedman MI, Norgren R, Scalera G, Seeley R (1995) Parabrachial nucleus lesions impair feeding response elicited by 2 , 5-anhydro-D-mannitol. Am J Phys 268:R676-R682

32. Allen MS, Bradford BJ, Oba M (2009) Board Invited Review: the hepatic oxidation theory of the control of feed intake and its application to ruminants. J Anim Sci 87:3317-3334

33. Berthoud HR, Kressel M, Neuhuber WL (1992) An anterograde tracing study of the vagal innervation of rat liver, portal vein and biliary system. Anat Embryol 186:431-442
34. Bernal-Mizrachi C, Xiaozhong L, Yin L et al (2007) An afferent vagal nerve pathway links hepatic PPARalpha activation to glucocorticoid-induced insulin resistance and hypertension. Cell Metab 5:91-102

35. Tsukita S, Yamada T, Uno K et al (2012) Hepatic glucokinase modulates obesity predisposition by regulating BAT thermogenesis via neural signals. Cell Metab 16:825-832

36. Uno K, Yamada T, Ishigaki Y et al (2015) A hepatic amino acid/mTOR/S6K-dependent signalling pathway modulates systemic lipid metabolism via neuronal signals. Nat Commun 6:7940

37. Yi CX, la Fleur SE, Fliers E, Kalsbeek A (2010) The role of the autonomic nervous liver innervation in the control of energy metabolism. Biochim Biophys Acta 1802:416-431

38. Obici S, Zhang BB, Karkanias G, Rossetti L (2002) Hypothalamic insulin signaling is required for inhibition of glucose production. Nat Med 8:1376-1382

39. Winnick JJ, Kraft G, Gregory JM et al (2016) Hepatic glycogen can regulate hypoglycemic counterregulation via a liver-brain axis. J Clin Investig 126:2236-2248

40. Xie H, Lautt WW (1996) Insulin resistance caused by hepatic cholinergic interruption and reversed by acetylcholine administration. Am J Phys 271:E587-E592

41. Matsuhisa M, Yamasaki Y, Shiba Y et al (2000) Important role of the hepatic vagus nerve in glucose uptake and production by the liver. Metab Clin Exp 49:11-16 